\title{
Analysis of a Model of Two Competitors in a Chemostat with an External Inhibitor
}

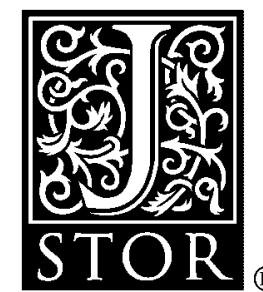

\author{
S. B. Hsu; P. Waltman
}

SIAM Journal on Applied Mathematics, Vol. 52, No. 2. (Apr., 1992), pp. 528-540.

Stable URL:

http://links.jstor.org/sici?sici=0036-1399\%28199204\%2952\%3A2\%3C528\%3AAOAMOT\%3E2.0.CO\%3B2-B

SIAM Journal on Applied Mathematics is currently published by Society for Industrial and Applied Mathematics.

Your use of the JSTOR archive indicates your acceptance of JSTOR's Terms and Conditions of Use, available at

http://www.jstor.org/about/terms.html. JSTOR's Terms and Conditions of Use provides, in part, that unless you have obtained prior permission, you may not download an entire issue of a journal or multiple copies of articles, and you may use content in the JSTOR archive only for your personal, non-commercial use.

Please contact the publisher regarding any further use of this work. Publisher contact information may be obtained at http://www.jstor.org/journals/siam.html.

Each copy of any part of a JSTOR transmission must contain the same copyright notice that appears on the screen or printed page of such transmission.

The JSTOR Archive is a trusted digital repository providing for long-term preservation and access to leading academic journals and scholarly literature from around the world. The Archive is supported by libraries, scholarly societies, publishers, and foundations. It is an initiative of JSTOR, a not-for-profit organization with a mission to help the scholarly community take advantage of advances in technology. For more information regarding JSTOR, please contact support@jstor.org. 


\title{
ANALYSIS OF A MODEL OF TWO COMPETITORS IN A CHEMOSTAT WITH AN EXTERNAL INHIBITOR*
}

\author{
S. B. HSU $\dagger$ AND P. WALTMAN $\ddagger$
}

\begin{abstract}
A model of the chemostat with an external nutrient and an external inhibitor is considered. A preliminary analysis reduces the problem to a three-dimensional competitive system. The theory of monotone flows is applied to obtain several global results. Global results fail when questions of multiple limit cycles cannot be answered. An example of an attracting limit cycle is given.

The chemostat with inhibitor can model competition between two populations of microorganisms, where one strain is resistant to an antibiotic or competition in detoxification, a system where one strain can take up the pollutant while the other is inhibited by it.
\end{abstract}

Key words. chemostat, inhibitor, contaminant, monotone dynamical system

AMS(MOS) subject classifications. $34 \mathrm{C} 35,92 \mathrm{~A} 17$

1. Introduction. In a recent paper, Lenski and Hattingh [ $\mathrm{LH}]$ considered a model for competition for a limiting resource in a chemostat between two populations in the presence of an inhibitor for one of the populations. An example might be two types of microorganisms, one of which is resistant to an agent also being input into the chemostat. The agent diminishes the growth rate of one population but is taken up by the other without ill effect. An example of such an agent is the nalidixic acid used in the experiments of Hansen and Hubbell $[\mathrm{HH}]$ to alter the growth rate of a strain of E. coli. The relevance to antibiotic-resistant or pesticide-resistant organisms is obvious. It is important to know when the resistant strain can out-compete the susceptible strain. A similar problem results with the desirability of the outcome reversed in a waste treatment or a detoxification problem. One strain can "detoxify" the environment, making it habitable to both.

The model (presented in $\S 2$ ) is essentially that of [ $\mathrm{LH}]$. By numerical computation, a variety of possible outcomes were shown, seven in all. Their approach was to fix the basic parameters and vary the input concentrations of the limiting nutrient and the inhibitor. Stability considerations were all local. Our approach is more mathematical. We attempt to find relationships among the parameters that classify the behavior types in a standardized (chemostat) environment. In many cases, we are able to give global results-precise theorems and proofs-that classify the possible behavior in terms of the parameters. To do this, it is convenient to scale out the influx concentrations, creating a standard (or relative) environment and deal with a reduced parameter set. We use the mathematical tools from nonlinear differential equations, particularly the theory of monotone dynamical systems, to construct a rigorous analysis of the behavior of solutions of the relevant nonlinear differential equations.

Finally, we note that we have found an interesting, additional outcome not listed in $[\mathrm{LH}]$ - the case of oscillatory coexistence. The system need not reach an equilibrium state for both competitors to survive.

2. The model. The model is that of a standard chemostat with two competitors, with the added feature that an inhibitor (toxicant) is also input from an external source.

\footnotetext{
* Received by the editors August 8, 1990; accepted for publication February 26, 1991.

${ }^{+}$Applied Mathematics Institute, National Tsing-Hua University, Hsinchu, Taiwan, Republic of China. This research was supported by the National Council of Science, Republic of China.

‡ Department of Mathematics, Emory University, Atlanta, Georgia 30322. This research was supported by National Science Foundation grant MCS-8901992.
} 
For basic results on the chemostat, see the survey articles [FS], [JM], [HET], [V], [WHH], and [W2]. One population is sensitive to the inhibitor, but the other takes it up without ill effect. The nutrient (and inhibitor) uptake and conversion (in the case of nutrient) is assumed to follow Michaelis-Menten dynamics. The results are probably valid for general monotone dynamics, but we were interested in providing explicit results, in terms of the measurable parameters, in hopes that such guidance might spur experimental effort, especially to demonstrate the oscillatory case.

Let $S(t)$ denote the nutrient concentration at time $t$ in the culture vessel; $x_{1}(t)$, $x_{2}(t)$, the concentration of the competitors; and $p(t)$, the concentration of the inhibitor (or toxicant or pollutant). The equations of the model take the form [LH]

$$
\begin{gathered}
S^{\prime}=\left(S^{(0)}-S\right) D-\frac{m_{1} x_{1} S}{a_{1}+S} f(p)-\frac{m_{2} x_{2} S}{a_{2}+S}, \\
x_{1}^{\prime}=x_{1}\left(\frac{m_{1} S}{a_{1}+S} f(p)-D\right), \\
x_{2}^{\prime}=x_{2}\left(\frac{m_{2} S}{a_{2}+S}-D\right), \\
p^{\prime}=\left(p^{(0)}-p\right) D-\frac{\delta x_{2} p}{K+p}, \\
S(0) \geqq 0, \quad x_{i}(0)>0, \quad p(0) \geqq 0, \quad i=1,2 .
\end{gathered}
$$

$S^{(0)}$ is the input concentration of the nutrient, and $p^{(0)}$ is the input concentration of the inhibitor, both of which are assumed to be constant. $D$ is the dilution rate of the chemostat. $S^{(0)}, p^{(0)}$, and $D$ are under the control of the experimenter. $m_{i}, a_{i}, i=1,2$ are the maximal growth rates of the competitors (without an inhibitor) and the Michaelis-Menten (or half saturation) constants, respectively. These parameters, inherent properties of the organism, are measurable in the laboratory. $\delta$ and $K$ play similar roles for the pollutant, $\delta$ being the uptake by $x_{2}$, and $K$ being a half saturation parameter. The function $f(p)$ represents the degree of inhibition of $p$ on the growth rate (or uptake rate) of $x_{1}$.

To reduce the number of parameters and to provide a standard environment so that comparisons can be made in terms of the parameters of the competing populations, the equations will be scaled. First, scale the units of concentration of $S, x_{1}, x_{2}$ by the input concentration $S^{(0)}$. This includes the parameters $a_{i}, i=1,2$. (We have already tacitly scaled out the yield parameters that scale the conversion of nutrient to organism.) Then scale time by the dilution rate (it has units $1 /$ time). This reduces $D$ to 1 and replaces $m_{i}$ by $m_{i} / D, i=1,2$, and $\delta$ by $\delta / D$. Finally, scale $p$ by $p^{(0)}$, which has the effect of scaling $p^{(0)}$ to 1 . In [LH], $f(p)=e^{-\lambda p}$; so this would be written as $\exp \left(-\lambda p^{(0)} p / p^{(0)}=\exp \left(-\lambda^{\prime} p\right)\right.$. If we make these changes and then, returning to the "old" names (for eample, using $m_{1}$ as the new "maximal growth rate," the "old $\left.m_{1} / D^{\prime \prime}\right)$, system (2.1) takes the form

$$
\begin{aligned}
& S^{\prime}=1-S-\frac{m_{1} x_{1} S}{a_{1}+S} f(p)-\frac{m_{2} x_{2} S}{a_{2}+S}, \\
& x_{1}^{\prime}=x_{1}\left(\frac{m_{1} S}{a_{1}+S} f(p)-1\right), \\
& x_{2}^{\prime}=x_{2}\left(\frac{m_{2} S}{a_{2}+S}-1\right),
\end{aligned}
$$




$$
\begin{gathered}
p^{\prime}=1-p-\frac{\delta x_{2} p}{K+p}, \\
S(0) \geqq 0, \quad x_{i}(0)>0, \quad i=1,2, \quad p(0) \geqq 0 .
\end{gathered}
$$

It is system (2.2) that will be investigated here. The reader is cautioned that the parameters have changed their meaning. They are to be viewed relative to the "standard environment" of $S^{(0)}=1, p^{(0)}=1, \tau=D t$ (the new nondimensional "time"). They are to be computed from the measured parameters and the operating parameters of the chemostat.

Concerning the function $f(p)$, we assume that (i) $f(p) \geqq 0, f(0)=1$, and (ii) $f^{\prime}(p)<0, p>0$. The function used in $[\mathrm{LH}]$ has these properties.

3. Preliminary analysis. In this section, model (2.2) is further simplified by noting that all trajectories of (2.2) are asymptotic to a particular set in $R^{3}$. This will make it sufficient to investigate a reduced set of equations. Moreover, certain cases are uninteresting in the current setting. These are identified, the behavior of trajectories analyzed, and the parameters restricted to exclude them in the remainder of the analysis. These results are global.

Let $\Sigma=1-S-x_{1}-x_{2}$. Then $\Sigma^{\prime}=-S^{\prime}-x_{1}^{\prime}-x_{2}^{\prime}=-1+S+x_{1}+x_{2}=-\Sigma$. System (2.2) may then be replaced by

$$
\begin{gathered}
\Sigma^{\prime}=-\Sigma, \\
x_{1}^{\prime}=x_{1}\left(\frac{m_{1}\left(1-\Sigma-x_{1}-x_{2}\right)}{a_{1}+1-\Sigma-x_{1}-x_{2}} f(p)-1\right), \\
x_{2}^{\prime}=x_{2}\left(\frac{m_{2}\left(1-\Sigma-x_{1}-x_{2}\right)}{a_{2}+1-\Sigma-x_{1}-x_{2}}-1\right), \\
p^{\prime}=1-p-\delta \frac{x_{2} p}{K+p} .
\end{gathered}
$$

Clearly, $\lim _{t \rightarrow \infty} \Sigma(t)=0$. Hence the solutions in the omega limit set of (3.1) must satisfy

$$
\begin{aligned}
& x_{1}^{\prime}=x_{1}\left(\frac{m_{1}\left(1-x_{1}-x_{2}\right)}{1+a_{1}-x_{1}-x_{2}} f(p)-1\right), \\
& x_{2}^{\prime}=x_{2}\left(\frac{m_{2}\left(1-x_{1}-x_{2}\right)}{1+a_{2}-x_{1}-x_{2}}-1\right), \\
& p^{\prime}=1-p-\delta \frac{x_{2} p}{K+p}, \\
& x_{i}(0)>0, \quad i=1,2, \quad p(0) \geqq 0, \quad x_{1}(0)+x_{2}(0)<1 .
\end{aligned}
$$

More directly, we could also apply the theory of asymptotically autonomous systems. See $[\mathrm{H}],[\mathrm{M}]$, and, for a detailed application, [HHW]. System (3.2) is competitive [Hi1], [S1], and we will make use of that theory when the need arises. Let

$$
\lambda_{1}=\frac{a_{1}}{m_{1}-1}, \quad \lambda_{2}=\frac{a_{2}}{m_{2}-1} .
$$

These are the usual parameters for the chemostat and would determine the outcome if the inhibitor $p$ were not present. The form of (3.2) guarantees that if $x_{i}(0)>0, i=1,2$, 
then $x_{i}(t)>0$ for $t>0$. Moreover, $\left.p^{\prime}\right|_{p=0}=1>0$; so, if $p(0) \geqq 0, p(t)>0$ for $t>0 . x_{1}(t)$ and $x_{2}(t)$ satisfy

$$
\begin{aligned}
& x_{1}^{\prime} \leqq x_{1}\left(\frac{m_{1}\left(1-x_{1}-x_{2}\right)}{1+a_{1}-x_{1}-x_{2}}-1\right), \\
& x_{2}^{\prime} \leqq x_{2}\left(\frac{m_{2}\left(1-x_{1}-x_{2}\right)}{1+a_{2}-x_{1}-x_{2}}-1\right),
\end{aligned}
$$

so that an application of Kamke's theorem, [C, p. 29] and the knowledge of the behavior of trajectories in the chemostat equations [HHW], [HSU], [W1] establishes the following proposition.

Proposition 3.1. If $m_{i} \leqq 1$ or if $\lambda_{i} \geqq 1, \lim _{t \rightarrow \infty} x_{i}(t)=0, i=1$ or 2 .

This simply states the biologically intuitive fact that if one of the competitors could not survive in the simple chemostat, that competitor will not survive in the chemostat with an inhibitor. Thus we may assume that $m_{i}>1$ and $0<\lambda_{i}<1, i=1,2$.

LEMMA 3.2. There exists a number $\gamma>0$ such that $p(t) \geqq \gamma$ for $t$ sufficiently large.

Proof. Suppose liminf $t_{t \rightarrow \infty} p(t)=0$. If $p(t)$ decreased to zero monotonically, then there would be a point $t_{0}$ such that for $t>t_{0}, p(t)+\delta p(t) /(K+p(t))<1$. For such values, $p^{\prime}(t)>0$, which contradicts $p(t)$ decreasing. Hence there exists a set of points $t_{n}, t_{n} \rightarrow \infty$, such that $p^{\prime}\left(t_{n}\right)=0$ and $p\left(t_{n}\right) \rightarrow 0$ as $t_{n} \rightarrow \infty$. For such values of $t_{n}$,

$$
\begin{aligned}
0 & =1-p\left(t_{n}\right)-\frac{\delta p\left(t_{n}\right) x_{2}\left(t_{n}\right)}{K+p\left(t_{n}\right)} \\
& >1-p\left(t_{n}\right)-\frac{\delta p\left(t_{n}\right)}{K+p\left(t_{n}\right)} \\
& >0
\end{aligned}
$$

for $n$ large. This establishes the lemma.

THEOREM 3.3. If $0<\lambda_{2} \leqq \lambda_{1}<1$, then

$$
\lim _{t \rightarrow \infty} x_{1}(t)=0, \quad \lim _{t \rightarrow \infty} x_{2}(t)=1-\lambda_{2}=x_{2}^{*}, \quad \lim _{t \rightarrow \infty} p(t)=p_{2}^{*}<1,
$$

where $p_{2}^{*}$ is the positive root of the quadratic

$$
(1-p)(K+p)-\delta\left(1-\lambda_{2}\right) p=0 .
$$

Proof. (The reason for labeling it $p_{2}^{*}$ will become clear in $\S 4$ ). $p_{2}^{*}<1$ follows from the fact that $p(t)$ satisfies

$$
p^{\prime}<1-p
$$

and the basic comparison theorem for differential inequalities. In view of Lemma 3.2, inequalities (3.4) can be replaced by

$$
\begin{aligned}
& x_{1}^{\prime} \leqq x_{1}\left(\frac{m_{1}\left(1-x_{1}-x_{2}\right) f(\gamma)}{1+a_{1}-x_{1}-x_{2}}-1\right), \\
& x_{2}^{\prime} \leqq x_{2}\left(\frac{m_{2}\left(1-x_{1}-x_{2}\right)}{1+a_{2}-x_{1}-x_{2}}-1\right),
\end{aligned}
$$

for $t$ sufficiently large. This system of inequalities can be compared to the equations 
for the chemostat with $\lambda_{2}$ and $\lambda_{\gamma}$ as parameters, where

$$
\lambda_{2} \leqq \lambda_{1}<\frac{a_{1}}{m_{1} f(\gamma)-1}=\lambda_{\gamma}
$$

so that the first component of the comparison system tends to zero as $t$ tends to infinity. Hence so does $x_{1}(t)$.

Thus, for the remainder of this paper, we may assume that

$$
m_{i}>1, \quad i=1,2 ; \quad 0<\lambda_{1}<\lambda_{2}<1
$$

to make the problem interesting. Note also that this provides the boundedness of solutions. The results in this section provide conditions for one or both of the competitors to wash out of the chemostat. To avoid "unlikely" cases, we tacitly assume that all rest points and periodic orbits are hyperbolic, i.e., that their stability is determined by their linearization.

4. The rest point set. As noted above, system (3.2) is competitive in the sense of Hirsch [Hi2]. This has a number of implications for the analysis. Foremost among these is that a type of Poincaré-Bendixson theory holds [S2], and the structure of attractors is severely limited [S1], [S2], [SW]. The only possible omega limit sets are those of a two-dimensional system, specifically a rest point, a periodic orbit, or a finite set of rest points connected by trajectories. Moreover, if there is a periodic orbit, it must have a rest point "inside," where "inside" is defined in terms of an order; see [S2] or [SW]. This has the consequence that when there is no interior rest point, there cannot be a periodic orbit in the open positive octant, and hence the limit is on the boundary. Thus the existence of an interior rest point is crucial for coexistence. These matters are discussed in more detail in $\S 5$, where some global results are presented. In this section, we concentrate on the existence and local stability of the rest point set. The following result will be helpful in the analysis below.

LEMMA 4.1 (Butler-McGehee lemma [FW, Lemma A1]). If $p$ is a hyperbolic rest point of (3.2) and is in the omega limit set $\omega$ of a trajectory $\gamma$, then either $\omega=\{p\}$ or there exist points $q_{1}, q_{2}$ in $\omega$, different from $p$, with $q_{1} \in M^{+}(p), q_{2} \in M^{-}(p) .\left(M^{+}\right.$and $M^{-}$denote the stable and unstable manifolds of $p$.)

See [BW], [BFW], and [DRS] for generalizations of this lemma.

There are three potential rest points on the boundary, which we label $E_{0}=(0,0,1)$, $E_{1}=\left(x_{1}^{*}, 0,1\right)$, and $E_{2}=\left(0, x_{2}^{*}, p_{2}^{*}\right)$. These correspond to one or both competitors becoming extinct.

$E_{0}$ always exists. $E_{2}$ exists with $x_{2}^{*}=1-\lambda_{2}$ and $p_{2}^{*}$ the root of (3.5) if $0<\lambda_{2}<1$, which is contained in our basic assumption (3.7). The existence of $E_{1}$ is a bit more delicate. In keeping with the definitions in (3.3), define $\lambda_{0}=a_{1} /\left(m_{1} f(1)-1\right)$. The inequality $0<\lambda_{0}<1$ corresponds to the survivability of the first population in a chemostat under maximal levels of the inhibitor. Easy computations show that $E_{1}=$ $\left(1-\lambda_{0}, 0,1\right)$ will exist if $\lambda_{0}>0$ and will have positive coordinates and be asymptotically stable in the $x_{1}-p$ plane if $0<\lambda_{0}<1$. If $1-\lambda_{0}$ is negative, $E_{1}$ is not meaningful, nor is it accessible from the given initial conditions since the $x_{2}-p$ plane is an invariant set. The stability of either $E_{1}$ or $E_{2}$ will depend on comparisons between the subscripted $\lambda$ 's. The local stability of each rest point depends on the eigenvalues of the linearization around those points. The Jacobian matrix for the linearization of (3.2) takes the form

$$
J=\left(\begin{array}{ccc}
m_{11} & m_{12} & m_{13} \\
m_{21} & m_{22} & 0 \\
0 & m_{32} & m_{33}
\end{array}\right) .
$$


At $E_{0}$,

$$
J=\left(\begin{array}{ccc}
\frac{m_{1} f(1)}{1+a_{1}}-1 & 0 & 0 \\
0 & \frac{m_{2}}{1+a_{2}}-1 & 0 \\
0 & -\frac{\delta}{1+K} & -1
\end{array}\right) .
$$

The eigenvalues are the diagonal elements. One eigenvalue is -1 , and the eigenvector lies along the $p$ axis. This corresponds to the growth of the inhibitor to its limiting value in the absence of a consumer. The set $\left\{\left.(0,0, p)\right|_{p>0}\right\}$ is invariant and is part of the stable manifold of $E_{0} \cdot m_{22}=\left(m_{2} /\left(1+a_{2}\right)\right)-1$ is positive since $\lambda_{2}<1$. Similarly, the remaining diagonal term $m_{11}$ is positive if $0<\lambda_{0}<1$, and negative otherwise. When this eigenvalue is negative, the stable manifold of $E_{0}$ is the entire $\left(x_{1}-p\right)$ plane.

Remark 4.2. When $\lambda_{0}>0$, no trajectory of (3.2) has $E_{0}$ as an omega limit point.

At $E_{1}, m_{21}=0$; since $m_{23}=m_{31}=0$, the eigenvalues are just the diagonal elements of $J$. Thus

$$
\mu_{1}=-\frac{m_{1} a_{1}\left(1-\lambda_{0}\right)}{\left(a_{1}+\lambda_{0}\right)^{2}} f(1), \quad \mu_{2}=\frac{\left(m_{2}-1\right)\left(\lambda_{0}-\lambda_{2}\right)}{a_{2}+\lambda_{0}}, \quad \mu_{3}=-1 .
$$

If $0<\lambda_{0}<\lambda_{2}<1$, then $E_{1}$ is asymptotically stable. This reflects the fact that $x_{1}$, in the presence of the maximal inhibitor concentration, is still a better competition than $x_{2}$. If $\lambda_{0}>\lambda_{2}, E_{1}$ is unstable and, of course, if $\lambda_{0}>1, E_{1}$ does not exist.

LEMMA 4.3. If $\lambda_{0}>\lambda_{2}$, then any solution of (3.2) satisfies $\liminf _{t \rightarrow \infty} x_{2}(t)>0$.

Proof. Suppose that Lemma 4.3 is not true. Then some trajectory $\Gamma$ has an omega limit point in the $\left(x_{1}-p\right)$ plane. Moreover, the initial conditions preclude that $\Gamma$ is on the stable manifold of $E_{1}$. Thus, by the Butler-McGehee lemma (Lemma 4.1), the omega limit set of $\Gamma$ must contain a point of the stable manifold of $E_{1}$ and hence the entire trajectory through that point. To remain bounded, such a trajectory must connect to $E_{0}$. We have already noted in Remark 4.2 that this is not possible.

At $E_{2}, m_{12}=m_{13}=m_{23}=0$, so again the eigenvalues are just the following diagonal elements:

$$
\begin{aligned}
& \mu_{1}=\frac{m_{1} \lambda_{2} f\left(p_{2}^{*}\right)}{a_{1}+\lambda_{2}}-1, \\
& \mu_{2}=-\frac{m_{2} a_{2}\left(1-\lambda_{2}\right)}{\left(a_{2}+\lambda_{2}\right)^{2}}, \\
& \mu_{3}=-1-\frac{\delta K\left(1-\lambda_{2}\right)}{\left(K+p_{2}^{*}\right)^{2}} .
\end{aligned}
$$

Clearly, $\mu_{2}$ and $\mu_{3}$ are negative, so $E_{2}$ always has a two-dimensional stable manifold. $\mu_{1}<0$ is equivalent to

$$
\lambda_{2}<\frac{a_{1}}{m_{1} f\left(p_{2}^{*}\right)-1}=\lambda^{*} .
$$

The local behavior of the rest point set on the boundary is summarized in Table 4.1, where $0<\lambda_{1}<\lambda_{2}<1$ is assumed. 
TABLE 4.1

\begin{tabular}{|c|c|c|}
\cline { 2 - 3 } \multicolumn{1}{c|}{} & Exists & Stability \\
\hline$E_{0}$ & always & 1- or 2-dimensional stable manifold \\
\hline$E_{1}$ & $0<\lambda_{0}<1$ & asymptotically stable if $0<\lambda_{0}<\lambda_{2}$ \\
\hline$E_{2}$ & $\lambda_{2}<1$ & asymptotically stable if $0<\lambda_{2}<\lambda^{*}$ \\
\hline
\end{tabular}

The more interesting case is that of an interior rest point. As noted above, the competitiveness of the system and a type of Poincaré-Bendixson theorem require its existence for coexistence to be possible. Let $E_{c}=\left(x_{1 c}^{*}, x_{2 c}^{*}, p_{c}^{*}\right)$ denote the coordinates of a possible interior rest point. First, it must be the case that

$$
1-x_{1 c}-x_{2 c}=\lambda_{2}
$$

for this is the only nontrivial zero of the derivative of $x_{2}$. Using this, we set the derivative of $x_{1}$ equal to zero to find that

$$
\frac{m_{1} \lambda_{2}}{a_{1}+\lambda_{2}} f(p)=1
$$

or that we need $\left(a_{1}+\lambda_{2}\right) / m_{1} \lambda_{2}$ to be in the range of $f(p)$. It it is, then

$$
p_{c}^{*}=f^{-1}\left(\frac{a_{1}+\lambda_{2}}{m_{1} \lambda_{2}}\right) .
$$

Since $f$ is monotone, this number is unique. Given $p_{c}^{*}$, then $x_{2 c}^{*}$ can be determined from setting $p^{\prime}(t)$ equal to zero, yielding

$$
1-p_{c}^{*}-\frac{\delta x_{2 c}^{*} p_{c}^{*}}{K+p_{c}^{*}}=0
$$

or

$$
x_{2 c}^{*}=\frac{\left(1-p_{c}^{*}\right)\left(K+p_{c}^{*}\right)}{\delta p_{c}^{*}} .
$$

This number is unique since $p_{c}^{*}$ is unique. If $x_{2 c}^{*}<1-\lambda_{2}$, then $x_{1 c}^{*}$ is uniquely determined from (4.2) as

$$
x_{1 c}^{*}=1-x_{2 c}^{*}-\lambda_{2} .
$$

Since $1-\lambda_{2}=x_{2}^{*}$, it follows that if $x_{2 c}^{*}$ exists, then $x_{2 c}^{*}<x_{2}^{*}$. This is the biologically expected statement that $x_{2}$ will do less well in the coexistent steady state than in the steady state where it is the sole survivor. This is true if and only if

$$
\dot{x}_{2 c}^{*}=\frac{\left(1-p_{c}^{*}\right)\left(K+p_{c}^{*}\right)}{\delta p_{c}^{*}}<\frac{\left(1-p_{2}^{*}\right)\left(K+p_{2}^{*}\right)}{\delta p_{2}^{*}}=x_{2}^{*}
$$

and hence, in view of the monotonicity of the expression in $p$, if and only if $p_{2}^{*}<p_{c}^{*}$. From (4.3) we have that this is equivalent to

$$
f\left(p_{2}^{*}\right)>\frac{a_{1}+\lambda_{2}}{m_{1} \lambda_{2}}
$$

or to the instability of $\left(E_{2}\right)$. See Table 4.1 , where the value of $\lambda^{*}$ has been substituted to obtain (4.6). Thus we have the following result. 
Proposition 4.4. If $\left(a_{1}+\lambda_{2}\right) / m_{1} \lambda_{2}$ is in the range of $f(p)$, then a necessary condition for the existence of an interior equilibrium for (3.2) is that $E_{2}$ exist and be unstable.

We see below that the interior equilibrium may exist even if $E_{1}$ does not. Before considering the stability of $E_{c}$, it remains to investigate whether (4.3) is feasible, to investigate whether $\left(a_{1}+\lambda_{2}\right) / m_{1} \lambda_{2}$ is in the range of $f(p)$.

If $0<\lambda_{0}<\lambda_{2}$, then $x_{1}$ is a better competitor than $x_{2}$ even at the maximal level of the inhibitor. A simple consequence of the definition of $\lambda_{0}$ is that $f(1)>\left(a_{1}+\lambda_{2}\right) / m_{1} \lambda_{2}$ in this case or that there is no value of $p_{c}^{*}, 0 \leqq p_{c}^{*} \leqq 1$, which satisfies (4.3). Hence $\lambda_{0} \geqq \lambda_{2}$ is a necessary condition for $\left(a_{1}+\lambda_{2}\right) / m_{1} \lambda_{2}$ to be in the range of $f(p), 0 \leqq p \leqq 1$. It is also sufficient since $f(0)=1$. Hence that Proposition 4.4 can be improved to $\lambda_{0} \geqq \lambda_{2}>\lambda^{*}$ is necessary and sufficient for the existence of $E_{c}$.

There remains the question of the stability of $E_{c}$. The matrix $J$ in (4.1) takes the form

$$
J=\left[\begin{array}{ccc}
-\frac{m_{1} a_{1}}{\left(a_{1}+\lambda_{2}\right)^{2}} f\left(p_{c}^{*}\right) x_{1 c}^{*} & -\frac{m_{1} a_{1}}{\left(a_{1}+\lambda_{2}\right)^{2}} f\left(p_{c}^{*}\right) x_{1 c}^{*} & \frac{m_{1} \lambda_{2}}{a_{1}+\lambda_{2}} x_{1 c}^{*} f^{\prime}\left(p_{c}^{*}\right) \\
-\frac{m_{2} a_{2}}{\left(a_{2}+\lambda_{2}\right)^{2}} x_{2 c}^{*} & -\frac{m_{2} a_{2}}{\left(a_{2}+\lambda_{2}\right)^{2}} x_{2 c}^{*} & 0 \\
0 & -\frac{\delta p_{c}^{*}}{K+p_{c}^{*}} & -1-\frac{\delta K x_{2 c}^{*}}{\left(K+p_{c}^{*}\right)^{2}}
\end{array}\right] .
$$

By expanding the determinant of $J$ in the last row, we see that it is negative or that the dimension of the stable manifold is one or three.

If $\delta<1$, the Gersgorin theory [LT, pp. 371, 376] immediately gives two roots with negative real parts-and hence three such roots-so we easily have that $E_{c}$ is asymptotically stable if $\delta<1$.

The characteristic roots of $J$ satisfy

$$
\begin{aligned}
\mu^{3} & +\mu^{2}\left(1+\frac{\delta K x_{2 c}^{*}}{\left(K+p_{c}^{*}\right)^{2}}+\frac{a_{1} x_{1 c}^{*}}{\left(a_{1}+\lambda_{2}\right) \lambda_{2}}+\frac{a_{2} x_{2 c}^{*}}{\left(a_{2}+\lambda_{2}\right) \lambda_{2}}\right) \\
& +\mu\left(1+\frac{\delta K x_{2 c}^{*}}{\left(K+p_{c}^{*}\right)^{2}}\right)\left(\frac{a_{1} x_{1 c}^{*}}{\left(a_{1}+\lambda_{2}\right) \lambda_{2}}+\frac{a_{2} x_{2 c}^{*}}{\left(a_{2}+\lambda_{2}\right) \lambda_{2}}\right) \\
& -\frac{f^{\prime}\left(p_{c}^{*}\right)}{f\left(p_{c}^{*}\right)} \frac{a_{2}}{\left(a_{2}+\lambda_{2}\right) \lambda_{2}} \frac{\delta p_{c}^{*}}{K+p_{c}^{*}} x_{1 c}^{*} x_{2 c}^{*}=0 .
\end{aligned}
$$

Since $f^{\prime}(p)<0$, the constant term is positive, so the Routh-Hurwitz criterion [C, p. 158] states that $E_{c}$ will be asymptotically stable if and only if

$$
\begin{aligned}
(1+ & \left.\frac{\delta K x_{2 c}^{*}}{\left(K+p_{c}^{*}\right)^{2}}+\frac{a_{1} x_{1 c}^{*}}{\left(a_{1}+\lambda_{2}\right) \lambda_{2}}+\frac{a_{2} x_{2 c}^{*}}{\left(a_{2}+\lambda_{2}\right) \lambda_{2}}\right)\left(1+\frac{\delta K x_{2 c}^{*}}{\left(K+p_{c}^{*}\right)^{2}}\right) \\
& \cdot\left(\frac{a_{1} x_{1 c}^{*}}{\left(a_{1}+\lambda_{2}\right) \lambda_{2}}+\frac{a_{2} x_{2 c}^{*}}{\left(a_{2}+\lambda_{2}\right) \lambda_{2}}\right)>-\frac{f^{\prime}\left(p_{c}^{*}\right)}{f\left(p_{c}^{*}\right)} \frac{a_{2}}{\left(a_{2}+\lambda_{2}\right) \lambda_{2}} \frac{\delta p_{c}^{*}}{K+p_{c}^{*}} x_{1 c}^{*} x_{2 c}^{*} .
\end{aligned}
$$

5. Dynamics without an interior rest point. If $E_{c}$ is not to exist, the inequality

$$
\lambda_{0} \geqq \lambda_{2}>\lambda^{*}
$$

must be violated. Recall that we are assuming that $0<\lambda_{1}<\lambda_{2}<1$ and, moreover, that $\lambda_{0}>\lambda^{*}$ holds by definition of these quantities and the monotonicity of $f(p)$. There are two possible outcomes depending on the way inequality (5.1) is violated.

Since system (3.2) is competitive, the possible dynamics are limited. Two results are of interest here [Hi1], [Hi2], [S2]. 
THEOREM 5.1 (See [S2, Thm. 2.2]). Let L be a compact omega limit set of an irreducible competitive system in $R^{3}$. If $L$ contains no equilibria, then $L$ is a closed orbit.

THEOREM 5.2 (See [S2, Thm. 2.4]). If $\gamma$ is a periodic orbit of an irreducible competitive system, then there exists at least one equilibrium in the "interior" of $\gamma$.

The term interior needs to be interpreted. It is the bounded component of the set

$$
K=\left(\gamma+R_{+}^{3}\right)^{c} \cap\left(\gamma-R_{+}^{3}\right)^{c},
$$

where $R_{+}^{3}$ is the positive cone in $R_{3}$, and the superscript $c$ denotes complement. See [S2] for more details. Since $m_{13}, m_{21}, m_{32}$ are nonzero in the interior of $R_{+}^{3},(4.1)$ is an irreducible matrix, so these results apply to system (3.2) there. We only make use of the following consequence in this section.

Remark 5.3. If $E_{c}$ does not exist, all omega limit sets lie on the boundary of $R_{+}^{3}$.

Since two-dimensional competitive dynamical systems have no periodic orbits, all omega limit sets contain equilibria. Moreover, there is at most one rest point in the interior of the $p-x_{1}$ and the $p-x_{2}$ (planar) faces. These observations will make the previously determined local stability results global.

THEOREM 5.4. If $0<\lambda_{1}<\lambda_{2}<\lambda^{*}$, then

$$
\lim _{t \rightarrow \infty} x_{1}(t)=0, \quad \lim _{t \rightarrow \infty} x_{2}(t)=x_{2}^{*}, \quad \lim _{t \rightarrow \infty} p(t)=p_{2}^{*} .
$$

Proof. $\lambda_{0}>0$ is implied by $\lambda^{*}>0$. If $\lambda_{0}>1$, then the only viable rest point is $E_{2}$ (Remark 4.2, Table 4.1), and it is locally asymptotically stable. If $\lambda_{0}<1$, then $E_{1}$ exists but is unstable and is not an omega limit point of a trajectory of (3.2). In either case, Remark 5.3 completes the proof.

THEOREM 5.5. If $0<\lambda_{0}<\lambda_{2}$ and $\lambda^{*}<\lambda_{2}$, then

$$
\lim _{t \rightarrow \infty} x_{1}(t)=x_{1}^{*}, \quad \lim _{t \rightarrow \infty} x_{2}(t)=0, \quad \lim _{t \rightarrow \infty} p(t)=1 .
$$

Proof. $E_{1}$ is locally asymptotically stable and $E_{2}$ is unstable. Remarks 4.2 and 5.3 complete the proof.

Since $\lambda^{*}>\lambda_{0}$ always holds, the above two theorems complete the asymptotic description of the dynamics under the basic hypothesis $0<\lambda_{1}<\lambda_{2}<1$ when there is no interior rest point. Note that $\S 3$ describes the cases where these inequalities do not hold.

6. Dynamics with an interior rest point. It was shown in $\S 4$ that a necessary condition for the existence of the interior equilibrium point $E_{c}$ was that $E_{2}$ be unstable. Ostensibly, there are three cases depending on $E_{1}$ :

(i) $E_{1}$ exists and is asymptotically stable,

(ii) $E_{1}$ exists and is unstable,

(iii) $E_{1}$ does not exist.

Case (i) does not occur, however, since (see Table 4.1) $E_{1}$ being asymptotically stable requires that $\lambda_{0}<\lambda_{2}$, and $E_{2}$ being unstable requires $\lambda_{2} \leqq \lambda^{*}$. However, from (4.3),

$$
f\left(p_{c}^{*}\right)=\frac{a_{1}+\lambda_{2}}{m_{1} \lambda_{2}}
$$

or

$$
\lambda_{2}=\frac{a_{1}}{m f\left(p_{c}^{*}\right)-1}<\frac{a_{1}}{m f(1)-1}=\lambda_{0},
$$

since $0<p_{2}^{*}<p_{c}^{*}<1$ and $f$ is decreasing. This contradicts $\lambda_{2}>\lambda_{0}$. Hence, we need to only consider cases (ii) and (iii).

The $n$-dimensional system $x^{\prime}=f(x)$ is said to be uniformly persistent if there exists a $\gamma>0$ such that

$$
\liminf _{t \rightarrow \infty} x_{i}(t) \geqq \gamma
$$


for $i=1,2, \cdots, n$. This subject has been extensively investigated in generality in the literature [FW], [BW], [BFW], [HW]; see [W3] for a brief survey or [HS] for a more complete exposition. The basic requirements are a dissipative semidynamical system, which leaves the boundary of the space invariant. Conditions are then imposed on the invariant sets on the boundary. In the simple case of (3.2), the sufficient conditions would be that the stable manifolds of the rest points on the boundary do not intersect the interior of the positive cone and that there is no cycle on the boundary, that is, a set of rest points and connecting orbits in the boundary that start and terminate at the same rest point.

THEOREM 6.1. If case (ii) or (iii) holds, then there exists a $\gamma>0$ such that every solution of (3.2) satisfies

$$
\liminf _{t \rightarrow \infty} x_{1}(t) \geqq \gamma, \quad \liminf _{t \rightarrow \infty} x_{2}(t) \geqq \gamma .
$$

Proof. To use the above-mentioned theorems on persistence, first, it is necessary to have a system of equations defined on an open region with a boundary. We use, instead of (3.2),

$$
\begin{gathered}
x_{1}^{\prime}=x_{1}\left(g_{1}\left(x_{1}+x_{2}\right) f(p)-1\right), \\
x_{2}^{\prime}=x_{2}\left(g_{2}\left(x_{1}+x_{2}\right)-1\right), \\
p^{\prime}=1-p-\frac{\delta x_{2} p}{K+|p|},
\end{gathered}
$$

where

$$
g_{i}(u)=\left\{\begin{array}{cl}
\frac{m_{i}(1-u)}{1+a_{i}-u}, & 0 \leqq u \leqq 1, \\
0, & u>1,
\end{array}\right.
$$

Since the only initial conditions of interest are with $p(0) \geqq 0, x_{i}(0)>0, x_{1}(0)+$ $x_{2}(0)<1$, solutions of these initial conditions are, for $t \geqq 0$, solutions of $(6.1)$. The open region is the wedge $x_{t}>0, i=1,2$ whose boundaries are the $x_{1}-p$ and $x_{2}-p$ planes in $R^{3}$, given by $x_{2}=0, x_{1} \geqq 0$ and $x_{1}=0, x_{2} \geqq 0$. The system is dissipative since in the extended region $x_{1}+x_{2}>1,\left(x_{1}(t)+x_{2}(t)\right)^{\prime}=-\left(x_{1}(t)+x_{2}(t)\right)$ and in the extended region $p<0, p^{\prime}(t) \geqq 1$. In cases (ii) or (iii), no portion of the stable manifolds of $E_{0}, E_{1}$, and $E_{2}$ intersect the interior of the wedge.

Moreover, since there is only one rest point interior to each of the $x_{1}-p$ and $x_{2}-p$ faces and an unstable rest point on the $p$ axis whose stable manifold is the $p$ axis, there are no connecting orbits to form a cycle. Hence, by Theorem 4.1 of [HW], (6.1) is uniformly persistent, and the theorem is established. (We could also use [BW], since the flow is on a locally compact space; using [HW], however, avoids the unpleasantness of trying to show the existence of backward orbits.)

For a three-dimensional competitive, uniformly persistent system with one interior rest point and with the positive cone invariant, a complete analysis of the possible limit sets was given in [SW]. However, system (3.2) does not fall directly within the scope of those results because it does not have an invariant positive cone and has difficulties for negative $p$. While negative $p$ is biologically unmeaningful, (3.2) makes sense for negative $p$ but is not a competitive system there. Rather than try to re-do [SW] for this particular case, we proceed to gather what information we can and refer the reader to [SW], especially Fig. 4.1, for intuitive purposes. Since system (3.2) is not competitive for $p<0$, the "cone" depicted in this figure extends "into" the noncompetitive region and does not terminate at a rest point as shown.

Theorem 6.1 guarantees the coexistence of both the $x_{1}$ and $x_{2}$ populations. However, it does not give the global asymptotic behavior. The further analysis of the 
system is complicated by the possibility of multiple limit cycles. Since this is a common difficulty in general two-dimension systems, it is not surprising that this presents difficulties in the analysis of three-dimension competitive systems.

TheOREM 6.2. Suppose that system (3.2) has no limit cycles. Then $E_{c}$ is globally asymptotically stable.

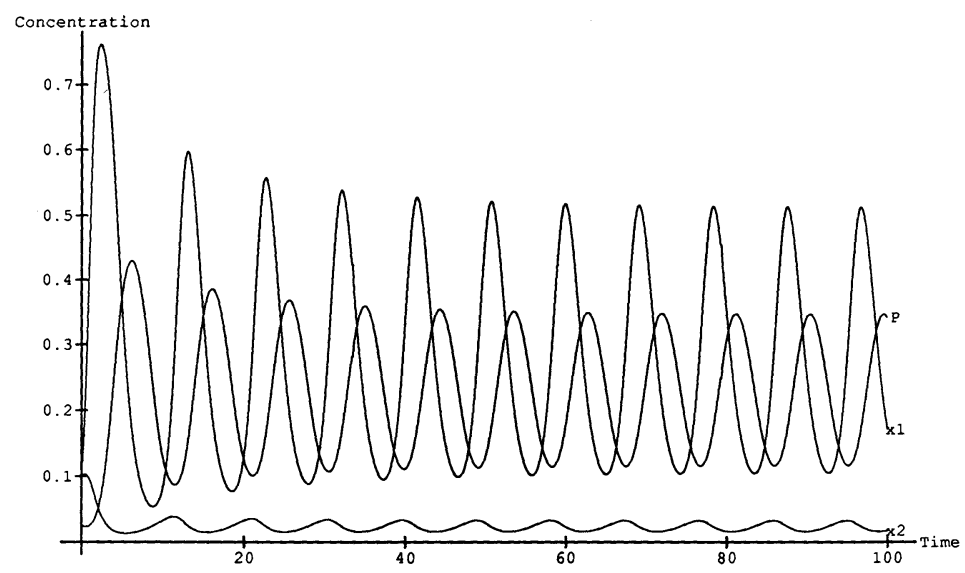

FIG. 6.1 Plot of 100 timesteps in the case of oscillatory coexistence. Parameters are $a_{1}=.5, a_{2}=3.5$, $m_{1}=5.0, m_{2}=6.0, \delta=50 ., K=.1, \eta=5.0$.

Proof. In view of Theorem 6.1, the omega limit set of any trajectory cannot be on the boundary $x_{1}=0$ or $x_{2}=0$. Away from the boundary, the system is irreducible. Since there are no limit cycles, all trajectories must tend to $E_{c}$ by Theorem 5.1 .

Conjecture. In case (ii), system (3.2) has no limit cycles.

We note that since we are assuming hyperbolicity, there must be at least two limit cycles for the conjecture to fail. Because of the assumed stability of $E_{c}$, there must be an unstable limit cycle with $E_{c}$ in its "interior" However, since the system is dissipative, there must be an asymptotically stable limit cycle as well.

THeOREM 6.3. Let $f(p)=e^{-\eta p}$ in (3.2) and let case (iii) hold. Then for $\eta$ sufficiently large, there exists $a \delta_{0}>0$ and a $K_{0}$, such that for $\delta>\delta_{0}$ and $K<K_{0}$, (3.2) has an attracting limit cycle.

Note that this behavior is not among those catalogued in [LH]. Figure 6.1 shows the time course for an example of this type of behavior. Figure 6.2 shows the limit cycle plotted in phase space.

Proof. The theorem follows if $E_{c}$ is unstable. To show this, we must show that (4.8) is violated. In the case under consideration, $-f^{\prime}(p) / f(p)=\eta$.

Define $c$ by $c=\ln \left(m_{1} \lambda_{2} /\left(a_{1}+\lambda_{2}\right)\right)$, and note that $c=\eta p_{c}^{*}$. It follows that

$$
1-p_{c}^{*}=1-\frac{c}{\eta}=\frac{(\eta-c)}{\eta} \text {. }
$$

Note that when $\eta$ is fixed, $p_{c}^{*}$ is fixed. From the definition of $x_{2 c}^{*}$ (see (4.4)), it follows that

$$
\frac{\delta K x_{2 c}^{*}}{\left(K+p_{c}^{*}\right)^{2}}=\frac{K}{K+p_{c}^{*}} \frac{1-p_{c}^{*}}{p_{c}^{*}}=\left(\frac{K}{K+p_{c}^{*}}\right)\left(\frac{\eta}{c}-1\right)
$$

for any choice of $\eta$ and the corresponding $p_{c}^{*}$. Fix $\eta$ satisfying

$$
\eta>c+2\left(3+\frac{a_{1}\left(1-\lambda_{2}\right)}{\left(a_{1}+\lambda_{2}\right) \lambda_{2}}\right)\left(\frac{a_{1}\left(1-\lambda_{2}\right)}{\left(a_{1}+\lambda_{2}\right) \lambda_{2}}+1\right)\left(\frac{2\left(a_{2}+\lambda_{2}\right) \lambda_{2}}{\left(1-\lambda_{2}\right) a_{2}}\right)
$$




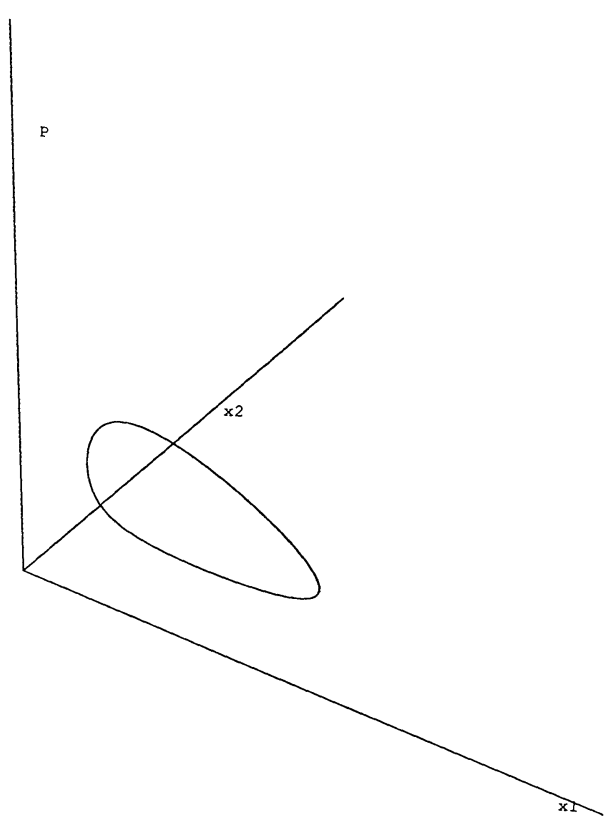

FIG. 6.2. Plot in $E^{3}$ of the limit cycle given in Fig. 6.1.

and $\left(m_{1} /\left(a_{1}+1\right)\right) e^{-\eta}<1$. Let $K_{0}$ be so small that the expression in (6.2) is less than 1. To show that (4.8) is violated, we estimate both sides. Note that, since $x_{1 c}^{*}=$ $1-\lambda_{2}-x_{2 c}^{*}, \lim _{\delta \rightarrow \infty} x_{2 c}^{*}=0$ and $\lim _{\delta \rightarrow \infty} x_{1 c}^{*}=1-\lambda_{2}$. Hence, for $\delta$ sufficiently large, $a_{2} x_{2 c}^{*} /\left(a_{2}+\lambda_{2}\right) \lambda_{2}<1$ and $1-\lambda_{2}>x_{1 c}^{*}>\left(1-\lambda_{2}\right) / 2$. The right-hand side of $(4.8)$ is bounded below by

$$
\frac{a_{2}}{2\left(a_{2}+\lambda_{2}\right) \lambda_{2}}\left(1-\lambda_{2}\right)(\eta-c) \text {. }
$$

The left-hand side of (4.8) has three factors, which we denote by $F_{1}, F_{2}, F_{3}$, respectively. By the discussion above, for $\delta$ sufficiently large,

$$
F_{1}<\left(3+\frac{a_{1}\left(1-\lambda_{2}\right)}{\left(a_{1}+\lambda_{2}\right) \lambda_{2}}\right), \quad F_{2}<2, \quad F_{3}<\frac{a_{1}\left(1-\lambda_{2}\right)}{\left(a_{1}+\lambda_{2}\right) \lambda_{2}}+1 .
$$

It follows that

$$
F_{1} F_{2} F_{3}<\frac{(\eta-c) a_{2}}{\left(a_{2}+\lambda_{2}\right) \lambda_{2}} \frac{\left(1-\lambda_{2}\right)}{2},
$$

which contradicts (4.8). Hence $E_{c}$ is unstable (with a two-dimensional unstable manifold). Choose a trajectory not on the stable manifold of $E_{c}$. By Theorem 5.1, its omega limit set must be a periodic orbit. By hyperbolicity, there must be an attracting orbit.

7. Discussion. This paper has considered competition for a single limiting nutrient in a simple chemostat with an inhibitor input in the feed bottle. The asymptotic behavior of this multidimensional model has been determined as a function of the basic parameters of the system. The results obtained are global in nature, except for the possibility of multiple limit cycles. The theory of dynamical systems played an important role in the analysis. Such classification theorems eliminate the need for exhaustive computer studies to determine the operating region of the chemostat.

The most unanticipated result was the presence of a stable limit cycle. Figures 6.1 and 6.2 illustrate this case. Figure 6.1 gives the time course, while Fig. 6.2 shows the 
limit cycle in the three-dimensional phase space. The theorem shows the possibility of limit cycles, but it is not known whether the parameters fall within the range of interest to biologists. Are there organisms that can coexist in this way? This result suggests a laboratory experiment that would be particularly important in the case of strains of competing bacteria where one is resistant to an antibiotic.

\section{REFERENCES}

[BW] G. Butler And P. Waltman, Persistence in dynamical systems, J. Differential Equations, 63 (1986), pp. 255-263.

[BFW] G. Butler, H. I. Freedman, And P. Waltman, Uniformly persistent systems, in Proc. Amer. Math. Soc., 96 (1986), pp. 425-430.

[C] W. A. Coppel, Stability and Asymptotic Behavior of Differential Equations, Heath, Boston, 1965.

[DRS] S. R. Dunbar, K. P. RYBAKowski, AND K. SCHMitT, Persistence in models of predator-prey populations with diffusion, J. Differential Equations, 65 (1986), pp. 117-138.

[FS] A. G. Frederickson And G. Stephanopoulos Microbial competition, Science, 213 (1981), pp. 972-979.

[FW] H. I. FREedmAN AND P. WALTMAN Persistence in models of three predator-prey populations, Math. Biosci., 68 (1984) pp. 213-231.

[H] J. K. HAle, Asymptotic Behavior of Dissipative Systems, Amer. Math. Soc., Providence, RI, 1988.

[HW] J. K. Hale AND P. Waltman Persistence in infinite-dimensional systems, SIAM J. Math. Anal., 20 (1989), pp. 388-395.

[HH] S. R. HANSEN AND S. P. HubBell, Single nutrient microbial competition; Agreement between experimental and theoretical forecast outcomes, Science, 20 (1980), pp. 1491-1493.

[HET] D. Herbert, R. Elsworth, And R. C. Telling, The continuous culture of bacteria: A theoretical and experimental study, J. Gen. Microbiol., 14 (1956), pp. 601-622.

[Hi1] M. HIRSCH, Systems of differential equations which are competitive or cooperative 1: Limit sets, SIAM J. Appl. Math., 13 (1982), pp. 167-179.

[Hi2] - The dynamical systems approach to differential equations, Bull. Amer. Math. Soc., 11 (1984), pp. 1-64.

[HS] V. Hutson And K. Schmitt, Permanence in dynamical systems, Math. Biosci., to appear.

[HHW] S. B. Hsu, S. P. HubBell, AND P. WALTMAN, A mathematical theory for single nutrient competition in continuous cultures of microorganisms, SIAM J. Appl. Math., 32 (1977), pp. 366-383.

[HSU] S. B. HsU, Limiting behavior for competing species, SIAM J. Appl. Math., 34 (1978), pp. 760-763.

[JM] H. W. JANNASH AND R. T. MATELES, Experimental bacterial ecology studies in continuous culture, Adv. Microbial Physiol., 11 (1974), pp. 165-212.

[LH] R. E. LENSKI AND S. HATTINGH, Coexistence of two competitors on one resource and one inhibitor: A chemostat model based on bacteria and antibiotics, J. Theoret. Bio., 122 (1986), pp. 83-93.

P. LANCASTER AND M. Tismenetsky, The Theory of Matrices, Academic Press, Orlando, 1985.

L. MARkus, Asymptotically autonomous differential systems, Contributions to the Theory of Nonlinear Oscillation, Vol. 3, Princeton University Press, Princeton, NJ, 1956, pp. 17-29.

H. SмIтH, Systems of ordinary differential equations which generate an order preserving flow: $A$ survey of results, SIAM Rev., 30 (1988), pp. 87-113.

- Periodic orbits of competitive and cooperative systems, J. Differential Equations, 65 (1986), pp. 362-373.

H. Smith And P. Waltman, A classification theorem for three dimensional competitive systems, J. Differential Equations, 70 (1987) pp. 325-332.

H. VeldCAmP, Ecological studies with the chemostat, Adv. Microbial Ecol., 1 (1977) pp. 59-95.

P. Waltman, S. P. Hubbell, And S. P. Hsu, Theoretical and experimental investigations of microbial competition in continuous culture, in Modeling and Differential Equations in Biology, Marcel Dekker, New York, 1980.

[W1] P. Waltman, T. Burton, ED., Competition Models in Population Biology Society for Industrial and Applied Mathematics, Philadelphia, PA, 1983.

[W2] - Competition in chemostat-like models, Rocky Mountain J. Math., 20 (1990), pp. $777-807$.

[W3] - A brief survey of persistence, in Delay Differential Equations and Dynamical Systems, S. Busenberg and M. Martelli, eds., Springer-Verlag, Berlin, New York, 1991, pp. 31-40. 
http://www.jstor.org

\title{
LINKED CITATIONS
}

- Page 1 of 2 -

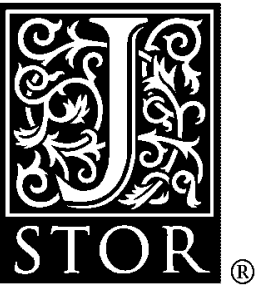

You have printed the following article:

\author{
Analysis of a Model of Two Competitors in a Chemostat with an External Inhibitor \\ S. B. Hsu; P. Waltman \\ SIAM Journal on Applied Mathematics, Vol. 52, No. 2. (Apr., 1992), pp. 528-540. \\ Stable URL: \\ http://links.jstor.org/sici?sici=0036-1399\%28199204\%2952\%3A2\%3C528\%3AAOAMOT\%3E2.0.CO\%3B2-B
}

This article references the following linked citations. If you are trying to access articles from an off-campus location, you may be required to first logon via your library web site to access JSTOR. Please visit your library's website or contact a librarian to learn about options for remote access to JSTOR.

\section{References}

\author{
${ }^{\mathrm{BFW}}$ Uniformly Persistent Systems \\ Geoffrey Butler; H. I. Freedman; Paul Waltman \\ Proceedings of the American Mathematical Society, Vol. 96, No. 3. (Mar., 1986), pp. 425-430. \\ Stable URL: \\ http://links.jstor.org/sici?sici=0002-9939\%28198603\%2996\%3A3\%3C425\%3AUPS\%3E2.0.CO\%3B2-I

\footnotetext{
${ }^{\mathrm{FS}}$ Metkephamid, a Systemically Active analog of Methionine Enkephalin with Potent Opioid \#-Receptor Activity

Robert C. A. Frederickson; Edward L. Smithwick; Robert Shuman; Kerry G. Bemis

Science, New Series, Vol. 211, No. 4482. (Feb. 6, 1981), pp. 603-605.

Stable URL:

http://links.jstor.org/sici?sici=0036-8075\%2819810206\%293\%3A211\%3A4482\%3C603\%3AMASAAO\%3E2.0.CO\%3B2-2
}

\section{${ }^{\mathrm{HH}}$ Single-Nutrient Microbial Competition: Qualitative Agreement between Experimental and Theoretically Forecast Outcomes}

Stephen R. Hansen; Stephen P. Hubbell

Science, New Series, Vol. 207, No. 4438. (Mar. 28, 1980), pp. 1491-1493.

Stable URL:

http://links.jstor.org/sici?sici=0036-8075\%2819800328\%293\%3A207\%3A4438\%3C1491\%3ASMCQAB\%3E2.0.CO\%3B2-O

NOTE: The reference numbering from the original has been maintained in this citation list. 
http://www.jstor.org

\section{LINKED CITATIONS \\ - Page 2 of 2 -}

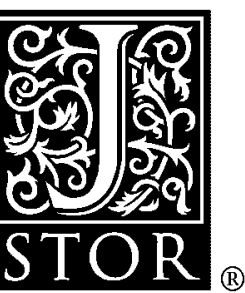

${ }^{\mathrm{HHW}}$ A Mathematical Theory for Single-Nutrient Competition in Continuous Cultures of Micro-Organisms

S. B. Hsu; S. Hubbell; P. Waltman

SIAM Journal on Applied Mathematics, Vol. 32, No. 2. (Mar., 1977), pp. 366-383.

Stable URL:

http://links.jstor.org/sici?sici=0036-1399\%28197703\%2932\%3A2\%3C366\%3AAMTFSC\%3E2.0.CO\%3B2-H

\section{${ }^{\mathrm{HSU}}$ Limiting Behavior for Competing Species}

S. B. Hsu

SIAM Journal on Applied Mathematics, Vol. 34, No. 4. (Jun., 1978), pp. 760-763.

Stable URL:

http://links.jstor.org/sici?sici=0036-1399\%28197806\%2934\%3A4\%3C760\%3ALBFCS\%3E2.0.CO\%3B2-I

${ }^{\mathrm{S} 1}$ Systems of Ordinary Differential Equations Which Generate an Order Preserving Flow. A Survey of Results

Hal L. Smith

SIAM Review, Vol. 30, No. 1. (Mar., 1988), pp. 87-113.

Stable URL:

http://links.jstor.org/sici?sici=0036-1445\%28198803\%2930\%3A1\%3C87\%3ASOODEW\%3E2.0.CO\%3B2-U

NOTE: The reference numbering from the original has been maintained in this citation list. 